\title{
Consommation alimentaire et apports nutritionnels chez les femmes allaitantes, en France
}

\author{
Pascale Hébel $^{1}$, Aurée Francou ${ }^{1}$, Louis Dominique Van Egroo ${ }^{2}$, Carole Rougé ${ }^{2,}$ et Pierre Mares $^{3}$ \\ ${ }^{1}$ CREDOC, 142 rue du Chevaleret, 75013 Paris, France \\ 2 Laboratoire Gallia, 383 rue Philippe Héron, 69400 Villefranche sur Saône, France \\ ${ }^{3}$ CHU Nîmes, Place du Pr. Robert Debré, 30029 Nîmes cedex 9, France
}

Reçu le 12 février 2018 - Accepté le 21 février 2018

\begin{abstract}
Résumé - Une bonne alimentation pendant la période d'allaitement est un facteur important pour la santé optimale des femmes et de leur enfant. Pour autant, aucune évaluation nutritionnelle n'a été effectuée sur cette population spécifique. L'objectif de cette étude est d'évaluer pour la première fois l'apport alimentaire et nutritionnel de femmes allaitantes vivant en France métropolitaine et d'évaluer son adéquation avec les recommandations locales. L'étude s'est déroulée entre septembre et octobre 2014 sur un échantillon de 250 femmes allaitantes. La majorité des participantes étaient âgées entre 25 et 34 ans (71,5\%), avaient un niveau d'étude supérieur (bac +1 ou plus) $(60,2 \%)$ et étaient mariées ou vivent maritalement $(94,9 \%)$. Les données de consommations alimentaires ont été recueillies avec un carnet de consommation alimentaire de 7 jours consécutifs. La majorité des participantes a renseigné le carnet sur 7 jours consécutifs $(88 \%)$. Le rythme alimentaire des participantes suit le modèle traditionnel français avec 3 repas principaux. Les participantes ingèrent en moyenne $2056 \pm 51,6 \mathrm{~g} / \mathrm{j}$ d'aliments et de boissons, dont plus de la moitié sous forme liquide $1168,4 \pm 42,6 \mathrm{~mL} / \mathrm{j}$. L'apport moyen en eau est de $617,6 \pm 36,5 \mathrm{~mL} / \mathrm{j}$. Un faible pourcentage de participantes a suivi les recommandations nutritionnelles en fruits et légumes, produits laitiers et produits céréaliers. L'apport énergétique moyen est de 1669,2 $\pm 32,8 \mathrm{kcal} / \mathrm{j}, 80 \%$ des femmes allaitantes sont en dessous des recommandations. L'apport en protéines représente $16 \%$ de l'apport énergétique total sans alcool. La moyenne totale d'apport de matières grasses s'élève à $66,2 \pm 1,5 \mathrm{~g} / \mathrm{j}$, valeur inférieure aux recommandations. L'apport en acides gras essentiels est inférieur aux recommandations pour la quasitotalité des femmes. L'étude suggère l'existence d'une inadéquation des apports alimentaires et nutritionnels et une faible observance des recommandations du PNNS et des Apports Nutritionnels Conseillés (ANC) chez des femmes allaitantes.
\end{abstract}

Mots clés : femme allaitantes / apports nutritionnels / consommation alimentaire / suivi des recommandations / allaitement

\begin{abstract}
Food and nutrient intake of lactating women, in France. Adequate nutrition during breastfeeding is an important factor to optimal health of women and their infant. As far as we can know, there has not been survey focused on Breastfeeding (Bf) women investigating their entire diet, and nutrient intakes as well as the adequacy of their food consumption to the PNNS guideline recommendations and of their nutrient intakes in relation to the French population reference intakes (PRIs). To evaluate the food and nutrient intakes of Bf women in France and to describe the compliance with the dietary and nutritional recommendations, a cross sectional survey was carried out in metropolitan France. Food intake was estimated through an online 7 day food diary. Two hundred and fifty Bf women were recruited from September 2014 and October 2014. Most of participants have filled the food dairy on 7 consecutive days $(88 \%)$. The traditional French model characterized by a daily dietary routine of three main meals continues in the study population. The participants consume an average of $2056 \pm 51.6 \mathrm{~g} / \mathrm{d}$ of food and beverages every day, half of which is in liquid form $(1168.4 \pm 42.6 \mathrm{~mL} /$ d). Mean water and total beverage intake were $617.6 \pm 36.5 \mathrm{~mL} / \mathrm{d}$ and $1168.4 \pm 42.6 \mathrm{~mL} / \mathrm{d}$, respectively. A low
\end{abstract}

\footnotetext{
*Correspondance : carole.rouge@danone.com
} 
proportion of participants met the French nutritional Guidelines (fruits and vegetables, dairy products, bread, cereals potatoes and legumes). Mean energy intake was $1669.2 \pm 32.8 \mathrm{kcal} / \mathrm{d}$, with $80 \%$ below the French recommendation. Mean protein intake represents $16 \%$ of total energy without alcohol. Mean total lipids intake was $66.2 \pm 1.5 \mathrm{~g} / \mathrm{d}$, below the PRI. Almost all mothers had the essential fatty acids intakes below the recommendations. The study suggests that $\mathrm{Bf}$ women may be at risk of food and nutrient inadequacies.

Keywords: lactating women / nutrient intake / food intake / adequacy to the recommandations / breastfeeding

\section{Introduction}

Pendant l'allaitement, l'alimentation et l'état nutritionnel de la mère sont clés pour contribuer à sa santé et à celle de son enfant. Il est rare que l'alimentation de la femme soit insuffisante aux besoins énergétiques de son enfant puisque le corps de la mère donne priorité aux besoins de l'enfant. L'alimentation de la mère qui allaite doit subvenir à ses besoins auxquels il faut rajouter les besoins liés à la lactation (Castel et Billeaud, 2017). Durant cette période, les besoins nutritionnels sont transitoirement augmentés et Castel et Billeaud (2017) préconisent une alimentation enrichie. Peu d'études de consommation alimentaire et nutritionnelle ont été réalisées chez les mères allaitantes. Les rares études publiées ont évalué la consommation d'un nutriment, tel que l'iode, le fer, les vitamines B et D et le statut nutritionnel associé chez cette population dans différents pays (Agarwal et al., 2006; Yang et al., 2014; Jones et al., 2007; Dawodu et al., 2015), ou ont examiné le degré d'adhésion des consommations alimentaires avec les recommandations (Ayatollahi, 2004; Sherwood et al., 2006). À notre connaissance, aucune enquête de consommations alimentaires n'a été réalisée chez la femme allaitante vivant en France. Seules existent des données chez la jeune femme adulte (Volatier, 2000; AFSSA, 2007; Hébel, 2004, 2012; InVS, 2007), rapportant une alimentation inadaptée (trop gras, trop sucré, pas assez de fruits et légumes...) associée à des apports nutritionnels inadéquats (vitamine B9, fer, zinc, iode...).

L'objectif de cette étude était d'évaluer pour la première fois les apports alimentaires et nutritionnels chez un échantillon de femmes allaitantes vivant en France métropolitaine, et de les comparer aux recommandations nutritionnelles françaises.

\section{Matériel et méthodes}

\subsection{Construction de l'échantillon}

Deux cent cinquante femmes allaitantes âgées entre 18 et 49 ans ont été recrutées entre septembre et octobre 2014 dans un panel Online en suivant des quotas d'âge et de niveau d'éducation. Les femmes interrogées ne devaient entretenir aucune relation proche avec des personnes travaillant dans les domaines suivants : fabrication ou vente au détail de produits alimentaires, communication, publicité, études de marché, instituts de sondage et journalisme. La TNS Sofres, organisme certifié pour la protection des données personnelles, a été chargée de recruter les femmes allaitantes. Elle a demandé l'accord libre et éclairé des femmes, en vue de leur participation à l'étude alimentaire. Toutes les femmes recrutées ont donné leur accord.

\subsection{Collecte des données}

Les informations relatives à la mère (âge, niveau d'étude, catégorie socio-professionnelle statut marital, parité, régions) et à l'enfant (sexe, âge, allaitement maternel exclusif et/ou mixte, nombre de tétées et/ou de biberons par jour, diversification alimentaire) ont été collectées par le biais d'un questionnaire auto-administré en ligne, lors du recrutement. En ce qui concerne les jumeaux, les données ont été collectées pour les deux enfants. Chaque participante a été invitée à se connecter à une plateforme en ligne afin de remplir un carnet alimentaire sur sept jours.

\subsection{1 Évaluation du régime alimentaire}

\subsubsection{Consommation d'aliments et de boissons}

Les participantes ont rempli un carnet alimentaire sur une période de 7 jours, selon une méthode similaire à celle appliquée pour d'autres études réalisées en France (Volatier, 2000; AFSSA, 2007; Hébel, 2004, 2012; InVS, 2007).

Chaque journée était répartie en 9 moments de consommation, à savoir les repas principaux et les périodes entre les repas. Pour chacun de ces moments, les participantes devaient indiquer l'heure de consommation des aliments, les différents produits alimentaires et boissons consommés et la quantité ingérée. En fonction du type d'aliment, les femmes interrogées devaient indiquer certaines caractéristiques de l'aliment, comme la marque ou la teneur réduite en sucre. Elles pouvaient indiquer si elles avaient sauté un repas. Les quantités d'aliments et de boissons consommées étaient estimées à l'aide du portionnaire photographique pour l'estimation de portions alimentaires SUVIMAX (2000). Elles pouvaient être également estimées directement en grammes ou en millilitres. Pour chaque aliment et chaque boisson, les participantes pouvaient indiquer leurs remarques et précisions dans un champ dédié.

Afin d'améliorer la qualité de la saisie des carnets alimentaires, les femmes interrogées devaient valider chaque journée de consommation pour indiquer qu'elles estimaient l'avoir correctement remplie. La validation de la journée nécessitait la saisie de 5 aliments ou boissons et le remplissage des trois repas principaux (petit-déjeuner, déjeuner et dîner, y compris si le repas avait été «sauté»). Le contrôle des validations de journées permettait un suivi plus pertinent des femmes qui étaient contactés par mail ou par téléphone quand les journées n'étaient pas toutes validées.

\subsubsection{Analyse alimentaire}

La consommation d'aliments et de boissons a été analysée pour 38 catégories alimentaires couvrant tous les apports (y compris 6 groupes pour les boissons), tels qu'utilisés précédemment en France (Hébel, 2007). À chaque aliment 
et boisson a été attribué un code nutritionnel de la base de données française de composition des aliments (CIQUAL, ANSES, 2013). Une attention particulière a été consacrée aux remarques et précisions des aliments indiquées par les femmes interrogées: elles apportaient des informations complémentaires sur les aliments ou les boissons concernées, ou notifiaient l'ajout d'aliments et des boissons supplémentaires. Les données manquantes sur les quantités ont été estimées par la valeur modale de l'échantillon pour chaque code nutritionnel. Un acte de consommation correspond à une prise alimentaire, identifiée par le code alimentaire et par l'heure de la journée à laquelle elle a été consommée. La portion correspond à la quantité consommée par acte de consommation.

\subsubsection{Analyse nutritionnelle}

Les apports en macronutriments, vitamines et minéraux provenant de l'alimentation ont été estimés sur la base des quantités consommées dans les carnets alimentaires et de la table de composition des aliments français CIQUAL (CIQUAL, ANSES, 2013).

\subsubsection{Critères d'exclusion}

Etant donné que l'équilibre alimentaire s'effectue sur la semaine, les carnets alimentaires de 3 jours ou d'une durée inférieure à 3 jours ont été exclus de l'analyse. Un apport énergétique moyen (sur la semaine d'enquête) supérieur à «moyenne +6 écart type » a été considéré comme aberrant et a été exclu. Les sous-déclarants ont été conservés dans l'analyse, conformément à l'avis de l'Autorité Européenne de Sécurité des Aliments (EFSA) (EFSA, 2009, 2014), afin d'éviter de créer un biais inconnu supplémentaire (Gibson, 2005).

\subsubsection{Scores d'adéquation du régime alimentaire}

La diversité du régime alimentaire a été définie, dans cette étude, comme le nombre des 38 groupes d'aliments consommés en l'espace de trois jours non consécutifs.

L'adéquation de l'apport nutritionnel a été analysée par rapport aux recommandations françaises pour les femmes allaitantes. Les Besoins Nutritionnels Moyens (BNM) sont définis comme $77 \%$ des apports nutritionnels conseillés de la population (ANC) publiés en France par Martin et al. (Martin, 2001) pour la population générale et pour certaines populations spécifiques, telles que les femmes allaitantes. Les ANC sont fixés par âge et sexe et pour les états physiologiques particuliers (croissance, grossesse, allaitement). Les recommandations utilisées dans cette étude sont celles spécifiquement fixées pour les femmes allaitantes.

L'adéquation aux recommandations alimentaires françaises du Programme National Nutrition Santé (PNNS, 20112015) pour les fruits et les légumes, le lait et les produits laitiers ainsi que le pain, les céréales, les pommes de terre et les légumes secs a été évaluée grâce au score PNNS-GS publié par Estaquio et al. (2009). Le score en continu (sur une échelle de dix) est construit de la façon suivante:

- un score de 0 est attribué à la non-consommation d'un nutriment ou d'un groupe d'aliments ;

- un score de 10 est attribué pour le suivi de la recommandation ;
- dans les autres cas, à savoir un apport inférieur au seuil minimum recommandé ou supérieur au seuil maximum recommandé, le score a été évalué comme suit :

- pour les apports inférieurs au seuil minimum recommandé : Score $=$ apport estimé/apport recommandé $\times 10$,

- pour les apports supérieurs au seuil maximum recommandé : Score $=$ apport recommandé/apport estimé $\times 10$.

Les portions de fruits et légumes, glucides et produits laitiers utilisées sont celles décrites dans l'étude ENNS (InVS, 2007). Les fruits et les légumes incluent les légumes seuls, les plats à base de légumes contenant au moins $50 \%$ de légumes, les soupes de légumes, les fruits seuls, les desserts contenant au moins $50 \%$ de fruits, ainsi que les jus de fruits et de légumes. Une portion représente $80 \mathrm{~g}$ de fruits/légumes ou $200 \mathrm{~g}$ de soupe ou $250 \mathrm{~mL}$ de jus de fruits/légumes. Au maximum, une portion de jus de fruits ou légumes est comptabilisé par jour. Les produits céréaliers incluent le pain et les biscottes, le pain utilisé pour les sandwiches, les pâtes, le riz, la farine, la semoule et les pommes de terre consommés seuls ou dans des plats cuisinés, les céréales pour le petit déjeuner et les légumes secs. Les produits laitiers incluent le yaourt et le lait fermenté, le fromage blanc, les petits suisses, les desserts laitiers contenant au moins $50 \%$ de lait, le fromage consommé seul (entier ou râpé), le lait consommé seul ou dans des boissons chaudes. Une portion est définie comme $30 \mathrm{~g}$ de fromage ou $125 \mathrm{~g}$ de yaourt ou $150 \mathrm{~mL}$ de lait.

\subsection{Analyse statistique}

L'échantillon de femmes allaitantes étudié a été redressé par rapport à la structure de la population des femmes en France publiée dans l'Enquête Nationale Périnatale de 2010 (Blondel et Kermarrec, 2011), sur les critères d'âge, de niveau d'éducation, de parité et du taux d'allaitement à 1 mois publié dans l'enquête EPIFANE 2012 (Salanave et al., 2012).

Les données de la consommation sont présentées dans les tableaux sous forme de moyenne, d'erreur standard à la moyenne, et d'écart-type à la moyenne. Le taux de consommation hebdomadaire est exprimé en pourcentage. Pour des jumeaux, les résultats sont présentés uniquement pour le premier enfant, tel que désigné par la mère. Le logiciel SAS 9.2 a été utilisé pour l'analyse statistique (SAS Institute Inc. Cary. NC.).

\section{Résultats}

\subsection{Population étudiée}

Quatre cent quatre-vingt-six femmes allaitantes ont été sollicitées pour participer à l'étude: 154 ne se sont jamais connectées à la plateforme dédiée à la saisie des données de consommation, 16 se sont connectées mais n'ont jamais renseigné le carnet de consommation. Parmi les 336 femmes allaitantes restantes, 65 ont été exclues car elles n'avaient pas renseigné le carnet alimentaire sur au moins 4 jours et 1 autre participante a été exclue car elle avait un apport énergétique aberrant. Au total, 250 participantes ont été inclues dans l'étude. Sur 250 carnets alimentaires analysés, $88 \%$ étaient renseignés sur 7 jours consécutifs, $4 \%$ sur 6 jours, $4 \%$ sur 5 jours et $4 \%$ sur 4 jours. 
Tableau 1. Caractéristiques relatives aux mères allaitantes en France participant à l'étude.

\begin{tabular}{|c|c|c|}
\hline$n=250$ & $n$ & $\%$ \\
\hline \multicolumn{3}{|l|}{ Tranche d'âge } \\
\hline $18-24$ ans & 17 & 7,0 \\
\hline $25-34$ ans & 179 & 71,5 \\
\hline $35-49$ ans & 54 & 21,5 \\
\hline \multicolumn{3}{|l|}{ Niveau d'études de la mère } \\
\hline Bac ou inférieur & 97 & 39,8 \\
\hline $\mathrm{Bac}+1$ ou plus & 151 & 60,2 \\
\hline \multicolumn{3}{|l|}{ Catégorie socio-professionnelle de la mère } \\
\hline $\begin{array}{l}\text { Agriculteur exploitant, artisan, commerçant, } \\
\text { chef d'entreprise }\end{array}$ & 9 & 4,1 \\
\hline Cadre, prof. intellec. sup., profession & 48 & 22 \\
\hline \multicolumn{3}{|l|}{ libérale } \\
\hline Employé & 46 & 21,5 \\
\hline Ouvrier & 100 & 46,4 \\
\hline Non concerné/inactif & 0 & 0 \\
\hline \multicolumn{3}{|l|}{ Statut marital } \\
\hline Célibataire & 10 & 3,9 \\
\hline Mariée ou vivant maritalement & 237 & 94,9 \\
\hline Divorcée ou séparée & 3 & 1,2 \\
\hline \multicolumn{3}{|l|}{ Parité } \\
\hline Primipare & 108 & 43,1 \\
\hline Multipare & 142 & 56,9 \\
\hline \multicolumn{3}{|l|}{ Régions } \\
\hline Nord & 21 & 8,4 \\
\hline Ouest & 33 & 13,1 \\
\hline Sud-Ouest & 27 & 11 \\
\hline Sud-Est & 64 & 25,5 \\
\hline Centre & 23 & 9,1 \\
\hline Est & 36 & 14,4 \\
\hline Région parisienne & 46 & 18,5 \\
\hline
\end{tabular}

Source: Enquête Femmes Allaitantes, CREDOC, 2016.

L'âge moyen des participantes était de $30,7 \pm 0,3$ ans. La majorité d'entre elles avaient un âge compris entre 25 et 34 ans $(71,5 \%)$, étaient mariées ou vivaient maritalement $(94,9 \%)$, avaient un niveau d'étude supérieure (Bac +1 ou plus) $(60,2 \%)$, étaient ouvrières ou employés - Cadres/Professions intellectuelles supérieures/Profession libérale (68,4\%), étaient multipares $(56,9 \%)$ et vivaient dans le Sud-Est, l'Est et la Région parisienne (58,4\%) (Tab. 1).

L'âge moyen du nouveau-né allaité au sein était de $5,3 \pm 0,2$ mois. Parmi les nouveau-nés, $52,9 \%$ étaient nourris exclusivement au sein, tandis que $41,7 \%$ recevaient également d'autres aliments solides ou liquides en plus du lait maternel ou du lait infantile. Le nombre moyen de consommations journalières de lait maternel était de 5,2 actes-soit sous la forme de tétée (5,6 fois par jour) soit sous la forme mixte de tétée et biberon de lait maternel (4,9 fois par jour).

\subsection{Rythme alimentaire}

Le rythme alimentaire quotidien observé dans la population étudiée est basé sur 3 repas principaux (petit-déjeuner,

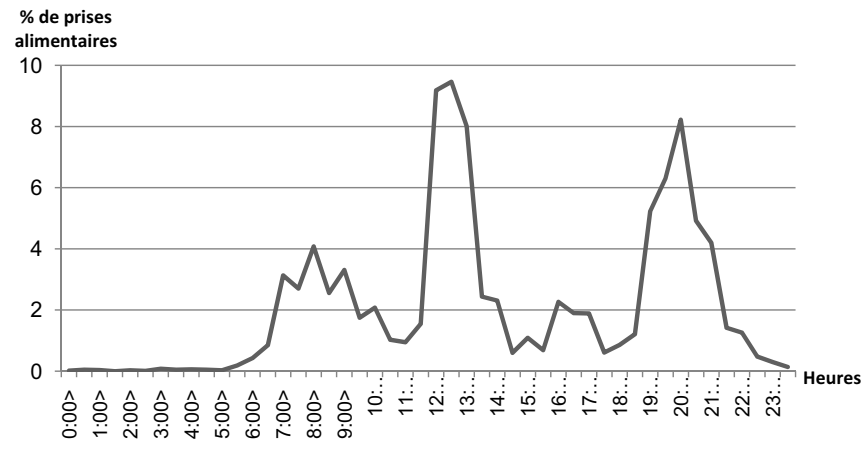

Fig. 1. Graphique de la répartition horaire des prises alimentaires chez les femmes allaitantes participant à l'étude. Base : 250 femmes allaitantes. Source: Enquête Femmes Allaitantes, CREDOC, 2016.

déjeuner et dîner) auxquels s'ajoutent les collations du matin et de l'après-midi (Fig. 1). Le modèle traditionnel français persiste dans la population étudiée.

Le déjeuner et le dîner représentaient respectivement $26,7 \%$ et $14,5 \%$ de l'apport journalier de nourriture, tandis que la consommation du petit-déjeuner et du goûter représentaient respectivement $15,8 \%$ et $6,1 \%$ de l'apport alimentaire journalier (Fig. 1).

\subsection{Consommations alimentaires et suivi des recommandations locales}

Les participantes ingèrent en moyenne $2056 \pm 51,6 \mathrm{~g} / \mathrm{j}$ d'aliments et de boissons, dont plus de la moitié sous forme liquide $1168,4 \pm 42,6 \mathrm{~mL} / \mathrm{j}$ (Tab. 2).

La majorité des participantes consomme de l'eau $(91,1 \%)$ avec un apport moyen journalier de $617,6 \pm 36,5 \mathrm{~mL} / \mathrm{j}$, inférieur aux recommandations locales. Une part importante des participantes consomme des jus et nectars $(63 \%)$ et des boissons sucrées $(61,2 \%)$ (Tab. 2). Parmi les participantes, $65,3 \%$ et $70,9 \%$ ont déclaré qu'elles consommaient régulièrement du café et du thé pendant la semaine.

Une minorité de la population étudiée $(21,1 \%)$ consomme des boissons alcoolisées au moins une fois par semaine, avec un apport journalier moyen de $54,5 \pm 6,8 \mathrm{~mL} / \mathrm{j}$ uniquement pour les consommatrices. Également, une minorité des participantes $(23 \%)$ consommait régulièrement du tabac.

La quasi-totalité des participantes $(98 \%)$ consomme des plats composés (plats transformés ou demandant peu de temps de préparation) avec un apport journalier moyen de $149,8 \pm 7,4 \mathrm{~g} / \mathrm{j}$ (Tab. 2). Cette catégorie d'aliment était la plus consommée.

La majorité des participantes consomme des légumes $(95,7 \%)$ avec un apport journalier moyen de $72,5 \pm 3,6 \mathrm{~g}$, tandis que les fruits sont consommés par $70,6 \%$ de la population étudiée, avec une consommation journalière moyenne de $60,2 \pm 5,3 \mathrm{~g}$.

La consommation moyenne en fruits et légumes était de $298,9 \pm 11,6 \mathrm{~g} / \mathrm{j}$ (Tab. 3). Seulement $11 \%$ des participantes consomment au moins 5 portions de fruits et légumes par jour. La majorité d'entre elles (74\%) consomme moins de 3,5 portions par jour. Leur consommation journalière moyenne était de 2,8 portions (dont 1,6 portion de légumes et 1,2 portion de fruits). Enfin, le score indicatif PNNS (PNNS-GS) était estimé en moyenne à 5,3 $\pm 0,2$ sur dix (Tab. 4). 
Tableau 2. Niveau moyen de consommation des différentes catégories d'aliments par la totalité de la population de l'étude et par les consommateurs uniquement de femmes allaitantes en France, sans prise en compte de l'apport nutritionnel des compléments alimentaires.

\begin{tabular}{|c|c|c|c|c|c|c|c|}
\hline \multirow{2}{*}{ Catégorie d'aliments } & \multicolumn{4}{|c|}{ Toutes les femmes allaitantes } & \multicolumn{3}{|c|}{ Consommatrices uniquement } \\
\hline & Moyenne & $\begin{array}{l}\text { Erreur } \\
\text { standard }\end{array}$ & Écart-type & $\begin{array}{l}\text { Taux de consommatrices } \\
\text { sur } 7 \text { jours }\end{array}$ & Moyenne & $\begin{array}{l}\text { Erreur } \\
\text { standard }\end{array}$ & Écart-type \\
\hline Total des aliments & 2056,6 & 51,6 & 816,5 & & 2056,6 & 51,6 & 816,5 \\
\hline Eaux & 617,6 & 36,5 & 576,5 & 91,1 & 677,9 & 37,8 & 570,1 \\
\hline Jus et nectars & 84,5 & 7,3 & 115,8 & 63,0 & 134,0 & 9,7 & 121,2 \\
\hline Boissons sucrées & 83,6 & 7,4 & 116,8 & 61,2 & 136,7 & 10,2 & 126,0 \\
\hline Légumes & 72,5 & 3,6 & 56,9 & 95,7 & 75,7 & 3,6 & 55,9 \\
\hline Produits laitiers & 65,6 & 4,4 & 68,8 & 87,0 & 75,4 & 4,6 & 68,2 \\
\hline Fruits & 60,2 & 5,3 & 83,5 & 70,6 & 85,3 & 6,5 & 86,6 \\
\hline Soupes & 44,2 & 4,3 & 68,1 & 43,7 & 101,2 & 6,6 & 69,3 \\
\hline Pizzas, quiches & 39,6 & 2,8 & 44,2 & 73,5 & 53,9 & 3,2 & 43,4 \\
\hline Pommes de terre ${ }^{a}$ & 39,2 & 2,5 & 39,1 & 80,7 & 48,6 & 2,7 & 38,3 \\
\hline Pâtisseries & 34,6 & 2,8 & 44,5 & 73,6 & 47,0 & 3,4 & 46,0 \\
\hline Desserts & 34,1 & 2,9 & 46,4 & 68,9 & 49,5 & 3,7 & 48,7 \\
\hline Viande & 33,6 & 1,9 & 30,7 & 77,7 & 43,3 & 2,0 & 28,4 \\
\hline Riz et semoule & 26,9 & 2,4 & 37,9 & 62,7 & 43,0 & 3,1 & 39,3 \\
\hline Sucreries & 21,5 & 1,4 & 22,8 & 89,1 & 24,1 & 1,5 & 22,6 \\
\hline Fromage & 20,8 & 1,3 & 21,1 & 82,5 & 25,2 & 1,4 & 20,6 \\
\hline Charcuterie & 20,3 & 1,5 & 23,6 & 75,8 & 26,8 & 1,7 & 23,8 \\
\hline
\end{tabular}

Aliments avec de faibles niveaux de consommation (consommation journalière $<20 \mathrm{~g} / \mathrm{j}$ en moyenne) non représentés dans le tableau : sandwiches, poisson et fruits de mer, viennoiseries, compotes et fruits au sirop, boissons alcoolisées, sauces, biscuits sucrés, œufs et produits dérivés, volailles et gibier, céréales pour le petit-déjeuner, légumes secs, matières grasses ajoutées, abats, biscuits salés, grains, condiments, fruits secs.

Source: Enquête Femmes Allaitantes, CREDOC, 2016.

a et apparentés (gnochis...).

Tableau 3. Nombre de portions journalières, fréquences et quantités des catégories d'aliments pour lesquelles le PNNS recommande une fréquence de consommation minimum chez les mères allaitantes en France.

\begin{tabular}{|c|c|c|c|c|c|c|c|c|c|}
\hline \multirow[b]{2}{*}{$\begin{array}{l}\text { Catégories } \\
\text { d'aliments PNNS } \\
\end{array}$} & \multicolumn{3}{|c|}{$\begin{array}{l}\text { Nombre de portions } \\
\text { /jour }\end{array}$} & \multicolumn{3}{|c|}{$\begin{array}{c}\text { Fréquences } \\
\text { Nombre de fois/jour }\end{array}$} & \multicolumn{3}{|c|}{$\begin{array}{l}\text { Quantités } \\
\text { g/jour }\end{array}$} \\
\hline & Moyenne & $\begin{array}{l}\text { Erreur } \\
\text { standard }\end{array}$ & $\begin{array}{l}\text { Écart } \\
\text { type }\end{array}$ & Moyenne & $\begin{array}{l}\text { Erreur } \\
\text { standard }\end{array}$ & $\begin{array}{l}\text { Écart } \\
\text { type }\end{array}$ & Moyenne & $\begin{array}{l}\text { Erreur } \\
\text { standard }\end{array}$ & $\begin{array}{l}\text { Écart } \\
\text { type }\end{array}$ \\
\hline Fruits et légumes & 2,8 & 0,1 & 1,8 & 1,2 & 0,0 & 0,5 & 298,9 & 11,6 & 182,8 \\
\hline Fruits & 1,2 & 0,1 & 1,2 & & & & & & \\
\hline Légumes & 1,6 & 0,1 & 1,0 & & & & & & \\
\hline Céréales & 2,3 & 0,1 & 1,2 & 2,2 & 0,0 & 0,5 & 218,6 & 6,3 & 100,4 \\
\hline
\end{tabular}

Source: Enquête Femmes Allaitantes, CREDOC, 2016.

La majorité des participantes consomme des produits laitiers $(87 \%)$ et du fromage $(82,5 \%)$ et près d'un tiers d'entres elles boivent du lait (32,6\%) (Tab. 2). La consommation moyenne de produits laitiers est de $213,1 \pm 9,9 \mathrm{~g} / \mathrm{j}$ (Tab. 3). Seulement 17,8 \% des participantes consomment entre 2,5 et 3,5 portions de produits laitiers par jour. La majorité d'entre elles $(84,5 \%)$ consomme moins de 3 portions par jour. Leur consommation journalière moyenne était de 2,1 portions de lait et de produits laitiers. Le score indicatif PNNS (PNNS-GS) était estimé en moyenne à 6,7 $\pm 0,2$ (Tab. 4).

Les produits céréaliers sont consommés par la majorité des participantes, en privilégiant notamment le pain/biscottes, les 
Tableau 4. Score Indicatif PNNS (PNNS-GS) applicable aux femmes allaitantes en France.

\begin{tabular}{|c|c|c|c|c|}
\hline \multirow[b]{2}{*}{ Recommandations } & \multirow[b]{2}{*}{$n$} & \multicolumn{3}{|c|}{ PNNS-GS (score sur 10) } \\
\hline & & Moyenne & $\begin{array}{l}\text { Erreur } \\
\text { standard }\end{array}$ & Écart type \\
\hline Lait et produits laitiers (entre 2,5 et 3,5 portions/jour) & 250 & 6,7 & 0,2 & 2,8 \\
\hline Pain, céréales, pommes de terre et légumes (à chaque repas : entre 3 et 6 portions/jour) & 250 & 6,9 & 0,2 & 2,5 \\
\hline
\end{tabular}

PNNS $=$ Programme National Nutrition Santé.

Source: Enquête Femmes Allaitantes, CREDOC, 2016.

pâtes, les pommes de terre, le riz et la semoule. La consommation moyenne de produits céréaliers est de $218,6 \pm 6,3 \mathrm{~g} / \mathrm{j}$. Seulement $20,8 \%$ des participantes consomment les 3 à 6 portions de produits céréaliers par jour. La majorité d'entre elles $(77 \%)$ consomme moins de 3 portions par jour. Leur consommation journalière moyenne était de 2,3 portions et le score indicatif PNNS (PNNS-GS) était estimé en moyenne à score PNNS était de 6,9 $\pm 0,2$ (Tab. 4).

Deux tiers des participantes consomment de la viande avec une consommation moyenne de 33,6 $\pm 3,9 \mathrm{~g} / \mathrm{j}$. Les abats, œufs et dérivés, les poissons et crustacés ainsi que les volailles et gibiers sont consommés plus rarement avec une consommation moyenne inférieure à $20 \mathrm{~g} / \mathrm{j}$.

La consommation de matières grasses ajoutées était limitée : $68,2 \%$ des participantes déclaraient en consommer, avec une consommation moyenne de $4,5 \pm 0,4 \mathrm{~g} / \mathrm{j}$.

Sur les 38 groupes d'aliments pris en compte dans l'étude, 17 représentent moins de $20 \mathrm{~g} / \mathrm{j}$ avec un taux de pénétration selon la catégorie d'aliments : entre $89,1 \%, 82,5 \%, 75,8 \%$ des femmes pour les produits sucrés, le fromage et la charcuterie, et $18,6 \%, 15,6 \%, 9 \%$ et $5,2 \%$ respectivement pour les biscuits salés et les légumes secs, les graines, les abats et les fruits secs (Tab. 2).

Enfin, la diversité alimentaire chez les femmes allaitantes est en moyenne de 9,7 groupes d'aliments consommés sur les 38 groupes d'aliments couvrant tous les apports sur trois jours.

\subsection{Apport énergétique et nutritionnel du régime alimentaire sans complément alimentaire}

L'apport énergétique moyen est de $1669,2 \pm 32,8 \mathrm{kcal} / \mathrm{j}$, $87 \%$ des femmes allaitantes se situant en dessous des recommandations françaises (Martin, 2001) (Tab. 5). La contribution moyenne des lipides, protéines, glucides au sein des apports énergétiques sans alcool (AESA) est respectivement de $35,8 \%, 16,4 \%$ et $47,8 \%$. Les glucides complexes et les fibres ne représentent que $22 \%$ et moins de $2 \%$ de l'AESA, respectivement. Les apports de glucides simples représentent $18,6 \%$ de l'AESA. Cette part importante des glucides simples dans l'apport énergétique est atteinte grâce à la consommation de jus de fruits et de nectars (4,1\% de l'AESA), à l'apport des boissons sucrées $(4,1 \%$ de l'AESA), à la consommation de fruits $(2,9 \%$ de l'AESA) et à l'apport relativement faible des produits et des biscuits sucrés $(1,0 \%$ et $0,5 \%$ de l'AESA).

Les apports moyens totaux de lipides, acide alphalinolénique, acide linoléique et acide docosahexaénoïque sont respectivement de $66,2 \pm 1,5 \mathrm{~g} / \mathrm{j}, 0,6 \pm 0,2 \mathrm{~g} / \mathrm{j}, 4,8 \pm 0,1 \mathrm{~g} / \mathrm{j}$ et
$0,1 \pm 0,0 \mathrm{~g} / \mathrm{j}$, avec un apport total en matières grasses inférieur aux ANC (Martin, 2001). La quasi-totalité des participantes présente un apport en acides gras essentiels inférieur aux recommandations (Martin, 2001) (Tab. 5). Enfin, la part moyenne des acides gras saturés, au sein des apports en lipides totaux est de $41,5 \%$.

Les apports moyens en minéraux, à savoir le calcium, l'iode et le zinc, sont inférieurs aux recommandations françaises (Martin, 2001) et même aux BNM en ce qui concerne l'iode et le zinc. Les apports moyens en magnésium, fer et phosphore sont supérieurs aux ANC (Martin, 2001). Les apports moyens en vitamines B1, B2, B3, B5, B6, B9, A, C, D et E sont inférieurs aux ANC (Martin, 2001) et aux BNM, sauf pour la vitamine B3 (Tab. 6).

\section{Discussion}

Notre étude a permis pour la première fois d'évaluer l'apport alimentaire et nutritionnel de femmes allaitantes vivant en France métropolitaine et d'évaluer son adéquation avec les recommandations locales.

\section{Consommations alimentaires}

Le modèle tradionnel français se caractérise par un rythme alimentaire quotidien basé sur trois repas principaux auxquels peut s'ajouter un goûter. Ce modèle persiste chez les femmes allaitantes étudiées.

Dans notre population d'étude, la consommation moyenne d'aliments et de boissons est de $2057 \mathrm{~g} / \mathrm{jour}$, dont plus de la moitié sous forme liquide $(1168 \mathrm{~g} / \mathrm{j})$. Ce rapport entre aliments solides et liquides est retrouvé chez les adultes issus de l'enquête nationale individuelle de consommations alimentaires (INCA 2) (AFSSA, 2007). Toutefois, la consommation moyenne d'aliments et de boissons observée est moins importante (2057 contre $2582 \mathrm{~g} / \mathrm{j}$ ) (AFSSA, 2007).

La consommation de liquides et notamment la consommation en eau, un élément clé de l'hydratation de l'organisme et de la lactation apparaît insuffisante $(\approx 618 \mathrm{~mL} / \mathrm{j})$ dans la population étudiée, en comparaison des apports adéquats de 1,5 L/j recommandés (PNNS, 2011-2015).

Notre étude suggère l'existence d'une inadéquation des apports alimentaires et une faible observance des recommandations du PNNS (2011-2015), vis-à-vis des fruits et légumes $(11 \%)$, des produits laitiers $(18 \%)$ et des produits céréaliers $(21 \%)$. 
Tableau 5. Apport moyen journalier énergétique et nutritionnel des mères allaitantes en France, et comparaison avec les apports de référence de la population.

Valeur moyenne Erreur standard Écart type BNM applicables aux femmes allaitantes Femmes avec apports $<$ BNM

\begin{tabular}{|c|c|c|c|c|}
\hline & & Kcal & & \\
\hline Énergie & 1669,2 & 32,8 & 518,2 & $2250^{\mathrm{a}}$ \\
\hline Énergie sans alcool & 1661,5 & 32,9 & 521,0 & \\
\hline
\end{tabular}

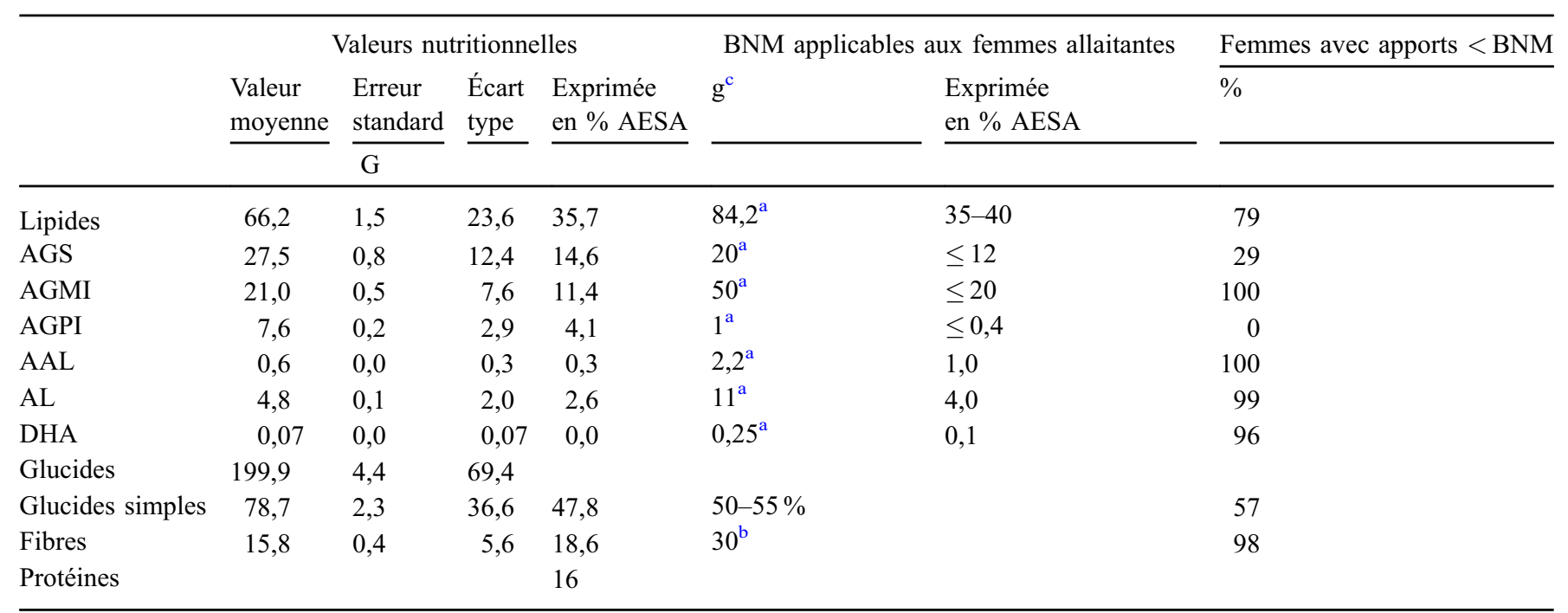

AAL, acide alpha-linolénique; DHA, acide docosahexaénoïque; AL, acide linoléique; AGMI, acides gras monoinsaturés ; AGPI, acides gras polyinsaturés; AGS, acides gras saturés; ANC, apports nutritionnels conseillés; AESA, apport énergétique sans alcool.

Source: Enquête Femmes Allaitantes, CREDOC, 2016.

a Recommandation spécifiquement applicable aux mères allaitantes.

b Recommandation applicable à la population générale.

c Sauf pour les glucides simples.

Tableau 6. Apports moyens en vitamines et minéraux des femmes allaitantes en France.

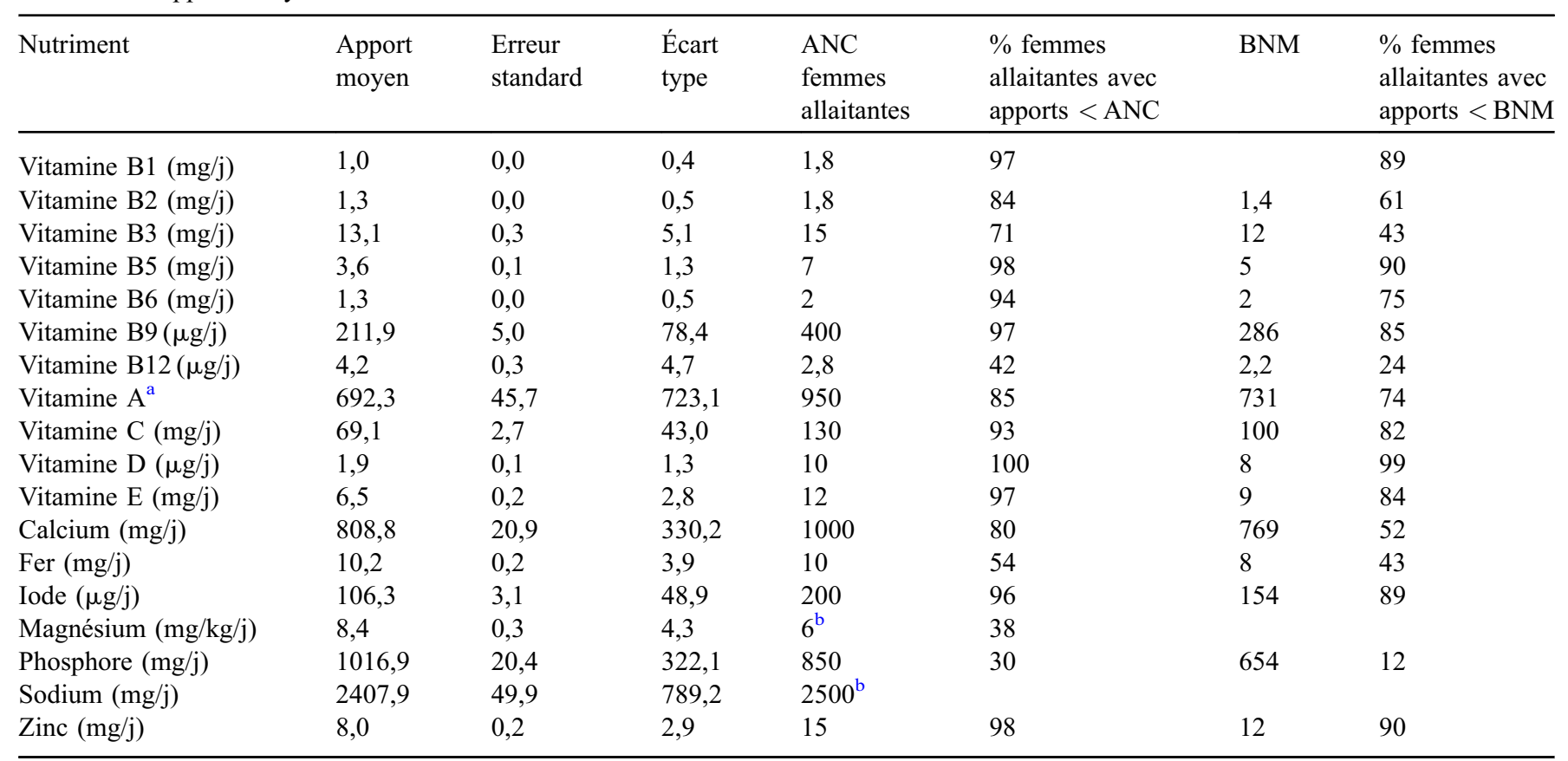

ANC, apports nutritionnels conseillés, BNM, besoins nutritionnels moyens.

Source: Enquête Femmes Allaitantes, CREDOC, 2016. 


\section{Apports énergétiques}

L'enquête nationale individuelle de consommations alimentaires (INCA2) (AFSSA, 2007) rapporte chez les femmes adultes, un apport énergétique total moyen de $1855 \mathrm{kcal} / \mathrm{j}$ et un apport énergétique sans alcool moyen de $1809 \mathrm{kcal} / \mathrm{j}$ (AFSSA, 2007). L'étude nationale nutrition santé de 2006 (InVS, 2007) a estimé l'apport énergétique total moyen à $1683 \mathrm{kcal} / \mathrm{j}$ et l'apport énergétique sans alcool (AESA) moyen à $1649 \mathrm{kcal} / \mathrm{j}$ chez la femme adulte. Dans notre population d'étude, l'apport énergétique total moyen estimé est de $1669 \mathrm{kcal} / \mathrm{j}$ et l'AESA moyen est de $1661 \mathrm{kcal} / \mathrm{j}$. Comme pour les quantités consommées, les enquêtes ne sont pas comparables. Toutefois, nous observons une tendance à un apport énergétique $(1669 \mathrm{kcal} / \mathrm{j})$ inférieur aux recommandations établies pour cette population spécifique $(2200 \mathrm{kcal} / \mathrm{j})$. Nous avons réalisé une analyse plus poussée des carnets alimentaires $(n=19)$ dont l'apport énergétique était inférieure ou égale à $1000 \mathrm{kcal} / \mathrm{j}$. Cette analyse a montré que cela concerne davantage les jeunes femmes (18-29 ans), à niveau d'études plus élevé (Bac +1 et plus), allaitant partiellement pour $70 \%$ d'entre elles. Ces femmes sont davantage consommatrices d'œufs, de poissons, volailles, de soupes, et moins consommatrices d'aliments à haute valeurs énergétiques (féculents, biscuits, viennoiseries, sucreries, sauces, sodas, jus et nectars, charcuteries, fromages, matières grasses ajoutées...). Ce profil de consommation correspond à celui des nouveaux comportements orthorexiques décrits par Denoux (2004) : « il s'agit de contrôler ce que l'on mange, croyant maîtriser ce que l'on est». L'orthorexie (addiction à la nourriture saine) est définie comme une fixation quasi pathologique sur la nourriture appropriée à laquelle on peut rattacher l'excès de consommation de légumes (soupes), de poissons, de viandes blanches. Denoux (2004) met en évidence l'influence de l'appareil législatif et réglementaire inflationniste sur le développement de cet effet pervers du danger imaginé par les orthorexiques. Le rapport des Français particulier à la nature serait aussi plus favorable à l'orthorexie : il est en effet plus difficile qu'ailleurs de supporter d'avoir peu d'emprise sur la nature. L'orthorexie est une façon de maîtriser les effets de l'environnement sur l'organisme. Robineau et de Saint Pol (2013) mettent en évidence la particularité culturelle de la France où la minceur est fortement valorisée par les femmes. La silhouette idéale, qui s'est amincie au cours du temps, conduit de plus en plus les jeunes femmes françaises à combiner idéal de la minceur et maîtrise de soi au travers de l'orthorexie qui se développe. Le profil des femmes allaitantes à faibles apports énergétiques (en dessous de $1000 \mathrm{kcal} / \mathrm{j}$ ) peut correspondre à un comportement récent des jeunes générations de femmes. Cette hypothèse nécessite d'être confirmée.

\section{Apports nutritionnels}

Notre étude suggère l'existence d'une inadéquation des apports nutritionnels (lipides, acides gras essentiels, acides gras saturés, glucides complexes, fibres, vitamines et minéraux) chez les participantes, en comparaison des apports nutritionnels conseillés applicables pour cette population spécifique (Martin, $2001)$ et des recommandations françaises (PNNS).

Les apports moyens en lipides et en acides gras essentiels (acide linoléique, acide alpha-linolénique et acide docosahé- xaénoïque) sont inférieurs aux recommandations applicables aux femmes allaitantes (ANC et BNM) (Martin, 2001), tandis que la part moyenne des acides saturés au sein des apports en lipides totaux est supérieure aux recommandations applicables à la population générale $(41,5 \%$ contre $36 \%$, [PNNS]).

La part moyenne des glucides complexes à l'AESA et l'apport moyen en fibres sont inférieurs aux recommandations applicables à la population générale, $(22 \%$ contre $>27,5 \%$, et $15,8 \mathrm{~g} / \mathrm{j}$ contre $30 \mathrm{~g} / \mathrm{j}$, respectivement [PNNS, 2011-2015; Martin, 2001]).

Les apports journaliers moyens en calcium, iode et zinc sont inférieurs aux recommandations applicables aux femmes allaitantes (ANC et BNM) (Martin, 2001). L'apport en calcium observée s'explique par une faible observance des recommandations du PNNS concernant la consommation de lait et de produits laitiers.

Les apports journaliers moyens en vitamines B1, B2, B3, B5, B6, B9, A, C, D et E sont inférieurs aux ANC et aux BNM, sauf pour la vitamine $\mathrm{B} 3$, ce qui peut être lié à une faible prévalence des participantes qui se sont conformées aux recommandations du PNNS en consommation de fruits et de légumes.

\section{Points forts de l'étude}

L'un des points forts de l'étude est l'utilisation d'un carnet alimentaire sur 7 jours pour l'évaluation de l'apport alimentaire, comme cela avait déjà été fait dans le cadre des études INCA, et dans les enquêtes alimentaires CCAF réalisées par le CREDOC sur la population française. Cette méthode est considérée adéquate par l'EFSA comme méthode principale d'évaluation à utiliser dans le cadre d'une enquête alimentaire pan-européenne (PNNS, 2011-2015). Néanmoins, le Groupe d'experts sur les données de consommation alimentaire mis en place par l'EFSA (EGFCD) a recommandé une durée maximale de 4 jours en ce qui concerne l'enregistrement des données par les participantes, en raison du manque de précision pouvant résulter, après cette période, de la lassitude de la participante (Black, 2000; Denoux, 2004). Nous avons utilisé à la fois des outils informatiques, des appels téléphoniques et des rappels par courrier par les diététiciens. Les portions ont été estimées par les femmes à l'aide du manuel-photos SUVIMAX, pour lequel une étude de validation des portions a été effectuée (Robineau et de Saint Pol, 2013).

\section{Limites de l'étude}

Une des limites principales de l'étude est que nous ne sommes pas en mesure d'identifier les non normo-déclarantes. Des futures études visant à évaluer le métabolisme de base des femmes allaitantes sont nécessaires. Les équations utilisées dans les méthodes d'identification ont besoin d'être revues en conséquence.

Une autre limite de l'étude est le mode de recrutement par Internet qui peut introduire un biais de sélection des participantes, favorisant les hauts niveaux d'études $(\mathrm{Bac}+1$ et plus) et les catégories socio professionnelles élevées et limitant les populations en situation de précarité ou de vulnérabilité sociale. 


\section{Conclusion}

L'étude suggère l'existence d'une inadéquation des apports alimentaires et nutritionnels, et une faible observance des recommandations du PNNS et des Apports Nutritionnels Conseillés chez des femmes allaitantes.

\section{Financement/aides}

Cette étude, réalisée par la TNS Sofres et le CREDOC, a été financée par Le Laboratoire Gallia.

Remerciements. Les auteurs remercient vivement la TNS Sofres pour sa contribution au recrutement des participantes à l'étude et Christine Bouley d'ORCHIDALI pour sa collaboration à la préparation et à la rédaction de cet article.

Conflits d'intérêts. Rougé C. et Van Egroo LD sont salariés du Laboratoire Gallia.

\section{Références}

AFSSA. 2007. Enquête individuelle et nationale sur les consommations alimentaires 2006-2007.

Agarwal KN, Agarwal DK, Sharma A, et al. 2006. Prevalence of anaemia in pregnant \& lactating women in India. Indian $J$ Med Res 124(2): 173-184.

Ayatollahi SM. 2004. Nutritional assessment of lactating women in Shiraz in relation to recommended dietary allowances. East Mediterr Health J 10(6): 822-827.

Black AE. 2000. The sensitivity and specificity of the Goldberg cutoff for EI: BMR for identifying diet reports of poor validity. Eur J Clin Nutr 54: 395-404.

Blondel B, Kermarrec M. 2011. Enquête nationale périnatale. Les naissances en 2010 et leur évolution depuis 2003. Inserm-U.953. Paris : Inserm, Drees, 132 p.

Castel B, Billeaud C. 2017. Alimentation de la mère allaitante. Cah Nutr Diet. Disponible sur https://doi.org/10.1016/j. cnd.2016.12.003.

CIQUAL, ANSES, 2013. French food composition table (https://pro. anses.fr/TableCIQUAL/). Consulté en novembre 2015.

Dawodu A, Davidson B, Woo JG, et al. 2015. Sun exposure and vitamin D supplementation in relation to vitamin D status of breastfeeding mothers and infants in the global exploration of human milk study. Nutrients 7(2): 1081-1093.

Denoux P. 2004. Nouvelles obsessions alimentaires: «l'orthorexie, une névrose culturelle?» Les actes du forum «aux bons soins de l'alimentation ». 14 décembre 2004, disponible sur http://www. agrobiosciences.org.

EFSA. 2009. General Principles for the collection of national food consumption data in the view of a pan-European dietary survey. EFSA J 7: 1435.

EFSA. 2014. Guidance on the EU Menu methodology. EFSA J 12: 3944.

Estaquio C, Kesse-Guyot E, Deschamps V, et al. 2009. Adherence to the French Programme National Nutrition Sante Guideline Score is associated with better nutrient intake and nutritional status. $J$ Am Diet Assoc 109: 1031-1041.

Gibson RS. 2005. Principles of Nutritional Assessment. 2nd édition. New York: Oxford University Press, 907 p.

Hébel P. (Coord). 2004. Comportements et consommations alimentaires en France. Paris : CREDOC, Tec \& Doc, Lavoisier.

Hébel P. (Coord). 2007. Comportements et consommations alimentaires en France. Paris : CREDOC, Tec \& Doc, Lavoisier.

Hébel P. (Coord). 2012. Comportements et consommations alimentaires en France. Paris : CREDOC, Tec \& Doc, Lavoisier.

InVS. 2007. Étude nationale nutrition santé ENNS (2006), situation nutritionnelle en France en 2006 selon les indicateurs d'objectif et les repères du Programme national nutrition santé (PNNS).

Jones KM, Ramirez-Zea M, Zuleta C, Allen LH. 2007. Prevalent vitamin B-12 deficiency in twelve-month-old Guatemalan infants is predicted by maternal B-12 deficiency and infant diet. $J$ Nutr 137(5): 1307-1313.

Martin A. 2001. Apports nutritionnels conseillés pour la population française. Paris : Édition Tec \& Doc, Lavoisier.

PNNS 2011-2015. Programme National Nutrition Santé. Disponible sur http://social-sante.gouv.fr/IMG/pdf/PNNS_2011-2015.pdf, Accessed March 08, 2016.

Robineau D, de Saint Pol T. 2013. Les normes de minceur: une comparaison internationale. Pop Soc INED 504.

Salanave B, De Launay C, Guerrisi C, Castetbon K. 2012. Taux d'allaitement maternel à la maternité et au premier mois de l'enfant. Résultats de l'étude Épifane, France. Bull Epidémiol Hebd 34: 383-387.

Sherwood KL, Houghton LA, Tarasuk V, O'Connor DL. 2006. Onethird of pregnant and lactating women may not be meeting their folate requirements from diet alone based on mandated levels of folic acid fortification. $J$ Nutr 136(11): 2820-2826.

SUVIMAX. 2000. Portions alimentaires: manuel photos pour l'estimation des quantités. Carnet photographique SUVIMAX. Paris : Polytechnica.

Volatier JL. 2000. Enquête individuelle et nationale sur les consommations alimentaires (INCA). Paris: Tec \& Doc, Lavoisier.

Yang J, Zheng H, Li X, et al. 2014. Assessment of iodine status and associated factors in vulnerable populations in Henan Province, China, in 2012. Asia Pac J Clin Nutr 23(4): 626-633.

Citation de l'article : Hébel P, Francou A, Egroo LDV, Rougé C, Mares P. 2018. Consommation alimentaire et apports nutritionnels chez les femmes allaitantes, en France. OCL 25(3): D303. 